\title{
Regulation of the Epithelial Sodium Channels by Small G-Proteins and Phosphatidylinositides
}

\author{
A. Staruschenko \\ Department of Physiology and Kidney Disease Center, Medical College of Wisconsin, Milwaukee, USA; \\ e-mail: staruschenko@mcw.edu
}

DOI: $10.1134 / \mathrm{S} 1990747809030465$

Epithelial $\mathrm{Na}^{+}$channels $(\mathrm{ENaC})$ determine sodium (re)absorption in distal nephron and also in other epithelial tissues. $\mathrm{ENaC}$ is a non-voltage gated highly selective sodium ion channel, which belongs to the $\mathrm{Deg} / \mathrm{ENaC}$ ion channel family. Genetic mutations leading to changing in channel function, cause electrolyte and water balance disturbances, and consequently to blood pressure regulation in the whole organism. It was previously shown that both small G-proteins and phosphatidylinositides participate in the management of different cell activity including signal transduction regulating the ion channel activity. We have demonstrated that small G-proteins of Ras, Rho and Rab families participate in $\mathrm{ENaC}$ regulation. We have shown that different small G-proteins activate $\mathrm{ENaC}$ through independent signal pathways and various mechanisms of action. The changes in ENaC activity by small G-proteins recruit phospholipids and its derivates. It was shown that small G-protein K-Ras affects ENaC through PI3-kinase increasing channel open probability. RhoA activates ENaC via Rho-kinase and PI(4)P 5- kinase, correspondingly. Activation of RhoA signaling pathway initiates a rapid channel translocation and integrating with plasma membrane. In both cases signal cascades initiated by small G-proteins cause the alteration of phosphatidylinositol-3,4,5-trisphosphate or phosphatidylinositol-4,5-bisphosphate (in the case of K-Ras and Rho-A, respectively). Those phosphatidylinositides consequently directly bind with the channel and lead to its activation. Furthermore, we recently showed that Rab small G-proteins that are responsible for vesicular transport are involved in $\mathrm{ENaC}$ regulation. We have identified two proteins in this family, Rab11a and Rab3a, which increase channel activity. We have determined that Rab11a colocalizes with $\mathrm{ENaC}$ and promotes the integration of the channel and plasma membrane. Mechanism of Rab3a activity is still unclear and demands further investigation.

Supported by American Heart Association (SDG 0730111N) and American Society of Nephrology (Carl W. Gottschalk Research Scholar Grant). 